\title{
Quark nuggets search using gravitational waves aluminum bar detectors
}

\author{
Francesco Ronga $^{\mathrm{a}}$ (ROG Collaboration) ${ }^{\mathrm{b}}$ \\ INFN Laboratori Nazionali di Frascati, Frascati, Italy
}

\begin{abstract}
Up to now there is no evidence of supersymmetric WIMPS dark matter. This may suggests to look for more exotic possibilities, for example compact ultra-dense quark nuggets. Nuclearites are an example of compact objects that could be constituent of the dark matter. After a short discussion on nuclearites, the result of a nuclearite search with the gravitational wave bar detectors NAUTILUS and EXPLORER is reported.
\end{abstract}

\section{Introduction}

During the last decade a very large experimental and theoretical effort has been devoted to understand the problem of dark matter (DM). Composite objects consisting of light quarks in a color super-conducting phase have been suggested as super-heavy quark nuggets DM; in addition, super-heavy DM anti-quark nuggets could exist and could perhaps solve the matterantimatter asymmetry [1]; the detection of such anti-quark nuggets by cosmic ray experiments is discussed in [2]. The energy loss predicted for super-heavy DM particles varies in different models, but it is likely that such particles could be confused with meteors, since the velocity, $270 \mathrm{~km} / \mathrm{sec}$, is in the high-end tail of the meteor velocity distribution. Here, we will focus our attention only on one possible kind of very massive particle called "nuclearite". It consists of neutral matter including a strange quark among its constituents. We make this choice because nuclearites are an example of particles already searched for by other experiments.

Nuggets of Strange Quark Matter (SQM), composed of approximately the same numbers of up, down and strange quarks could be the true ground state of quantum chromodynamics [3].

According to [4] nuclearites are considered to be large strange quark nuggets, with overall neutrality ensured by an electron cloud which surrounds the nuclearite core, forming a sort of atom. Nuclearites with galactic velocities are protected by their surrounding electrons against coulomb interactions with the atoms they might hit.

As a consequence, the principal energy-loss mechanism for a nuclearite passing through matter is atomic collision. For a massive nuclearite the energy-loss rate is:

$$
\frac{d E}{d x}=-A \rho v^{2}
$$

\footnotetext{
a e-mail: francesco.ronga@lnf.infn.it

${ }^{b}$ ROG ollaboration: M. Bassan, E. Coccia, S. D’Antonio, V. Fafone, G. Giordano, A. Marini, Y. Minenkov, I. Modena, G.V. Pallottino, G. Pizzella, A. Rocchi, F. Ronga, M. Visco.
} 
where $\rho$ is the density of the traversed medium, $v$ the nuclearite velocity and $A$ is its effective cross-sectional area. The effective area can be obtained by the nuclearite density $\rho_{N}$. For a small nuclearite of mass less than $1.5 \mathrm{ng}$, the cross-section area $A$ is controlled by its electronic atmosphere which is never smaller than $10^{-8} \mathrm{~cm}: A=\pi 10^{-16}$ for $M<1.5 \mathrm{ng}$ otherwise $A=\pi\left(3 M /\left(4 \pi \rho_{N}\right)^{2 / 3}\right.$ where $\rho_{N}=3.5 \cdot 10^{14} \mathrm{~g} / \mathrm{cm}^{3}$ is the nuclearite density and $M$ its mass. According to Eq. (1), nuclearites having galactic velocity and mass heavier than $10^{-14} \mathrm{~g}$ penetrate the atmosphere, while those heavier than $0.1 \mathrm{~g}$ pass freely though an Earth diameter. Equation (1) has been used by [4] to compute the amount of visible light emitted in the atmosphere, assuming that the light is emitted as a black-body radiation from an expanding cylindrical thermal shock wave and to compute therefore the apparent magnitude as defined for meteors. It turns out that there are two important differences that can help to discriminate between nuclearites and meteors. The first one is that the amount of light emitted by nuclearites is proportional to the atmospheric density and therefore most of the light is emitted near the ground level; the second difference is that a nuclearite of mass bigger than $0.1 \mathrm{~g}$ can move upward and this is extremely unlikely for a meteor.

The nuclearite search could be an interesting by-product of cosmic ray space experiments looking from space to the light produced in the atmosphere. The possibility to study meteors and to search nuclearites and other quark nuggets in the planned JEM-EUSO mission has been studied in Ref. [5].

Nuclearites and similar particles have been searched using different approaches. Many techniques have been used to detect nuclearites: acoustic emission due to the thermal shock in aluminum gravitational wave cylindrical detectors, damages in plastic materials like CR39, Makrofol or Lexan, light emission in oil or sea water, seismic waves induced by big nuclearites. Due to the uncertainties in the conversion of the energy losses in a measurable signal it is important to have different techniques to detect such exotic particles. Figure 1 shows upper limits of a representative experiment of each technique. It is not aimed at being a full list of the experiments done so far to search nuclearites, but it is a reasonable summary of the state of the art in this field. The lower line represents the JEM-EUSO possible upper limit in $24 \mathrm{~h}$.

\section{The NAUTILUS and EXPLORER gravitational wave bar detectors used as particle detector}

The gravitational wave ( $g w$ ) detector NAUTILUS [10] is located in Frascati (Italy) National Laboratories of INFN. NAUTILUS started operations around 1998. The current run started in 2003. The detector EXPLORER, similar to NAUTILUS, was located in CERN (Geneva-CH). The EXPLORER run ended in June 2010.

Both detectors use the same principles of operation. EXPLORER and NAUTILUS consist of a large aluminum alloy cylinder ( $3 \mathrm{~m}$ long, $0.6 \mathrm{~m}$ diameter) suspended in vacuum by a cable around its central section to reduce the seismic and acoustic noise and cooled to about $2 \mathrm{~K}$ by means of a superfluid helium bath. The $(g w)$ excites the odd longitudinal modes of the cylindrical bar. To record the vibrations of the bar first longitudinal mode, an auxiliary mechanical resonator tuned to the same frequency is bolted on one bar end face. This resonator is part of a capacitive electro-mechanical transducer that produces an electrical a.c. current that is proportional to the displacement between the secondary resonator and the bar end face. Such current is then amplified by means of a dcSQUID superconductive device and recorded on disk with an ADC sampled at $5 \mathrm{kHz}$.

Both detectors are equipped with cosmic ray telescopes, to veto excitations due to large showers. The two telescopes rely on different technologies (scintillators for Explorer, 


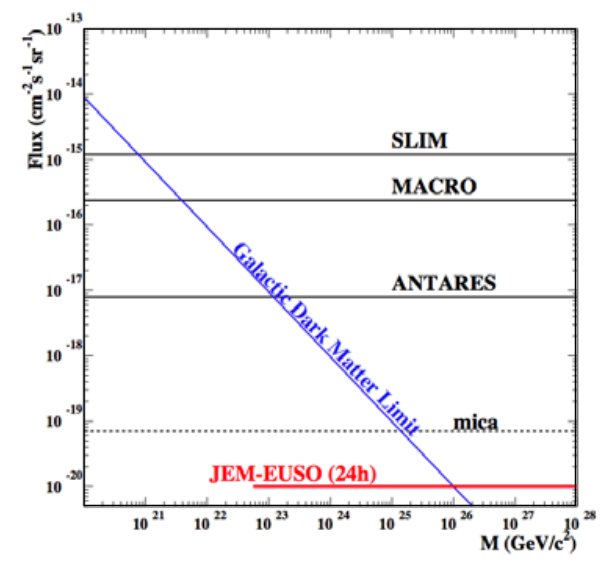

Figure 1. The 90\% confidence level upper limit on the flux of nuclearites of experiments with different techniques $[7-9,12]$. The "old" mica limits [12] are dependent from several additional assumptions, respect to the other experiments. The lower line shows the possible limit in $24 \mathrm{~h}$ of the JEM-EUSO cosmic ray experiment planned for the international space station [5]. Note that most of the proposed quark nuggets have energy losses bigger than the neutral nuclearites. Therefore limits applies to many other candidates.

streamer tubes for NAUTILUS) but both provide a monitor of comparable performances and a continuous check of the antenna sensitivity. The data are processed off-line, applying adaptive frequency domain filters optimized for delta-like signals. The noise characteristics estimate is updated averaging the output over 10 minutes periods. Traditionally the noise is expressed as energy in Kelvin units.

The interaction of energetic charged particles with a normal mode of an extended elastic cylinder has been extensively studied over the years, both on the theoretical and on the experimental side [11].

According the so called "thermo acoustic model" a particle crossing the bar produces longitudinal vibrations originated from the local thermal expansion due to the warming up due to the energy loss. In particular, the vibration amplitude is directly proportional to the ratio of two thermo-physical parameters of the material, namely the thermal expansion coefficient and the specific heat at constant volume. The ratio of these two quantities appears in the definition of the Grüneisen parameter $\gamma$.

The vibrational energy $E$ of the fundamental vibrational mode due the energy loss of a particle crossing an aluminum cylindrical bar is [11]:

$$
E=\frac{4}{9 \pi} \frac{\gamma^{2}}{\rho L v^{2}}\left(\frac{d W}{d x}\right)^{2} \times\left[\sin \left(\frac{\pi z_{o}}{L}\right) \frac{\sin \left[\left(\pi l_{o} \cos \left(\theta_{o}\right) / 2 L\right)\right]}{\pi R \cos \left(\theta_{o}\right) / L}\right]^{2}
$$

where $L$ is the bar length, $R$ the bar radius, $l_{o}$ the length of the particle track inside the bar, $z_{o}$ the distance of the track mid point from one end of the bar, $\theta_{o}$ the angle between the particle track and the axis of the bar, $\frac{d W}{d x}$ the energy loss of the particle in the bar, $\rho$ the density, $v$ the longitudinal sound velocity in the material. This relation is valid for the normal-conducting (n) state material and some authors (see the references in [11]) have extended the model to a super-conducting $(s)$ resonator, according to a scenario in which the vibration amplitude is due to two pressure sources, one due to $s-n$ transitions in small regions centered around the interacting particle tracks and the other due to thermal effects in these regions now in the $n$ state. 


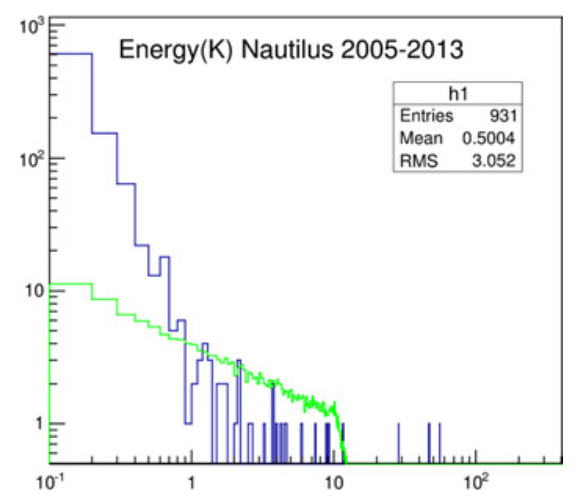

Figure 2. Vibrational energy distribution of the NAUTILUS events, after the cuts discussed in the text. The lower plot (in green) is the result of a Montecarlo simulation for $\beta \theta(M)=0.004$ and is normalized to data.

It is important to note, at this point, that a $g w$ bar antenna, used as particle detector, has characteristics very different from the usual particle detectors which are sensitive to ionization losses: indeed an acoustic resonator can be seen as a zero threshold calorimeter, sensitive to a vast range of energy loss processes. The usual $g w$ software filter works well up to a time scale of the order of the order of $5 \mathrm{msec}$, corresponding to a $\beta=4 \times 10^{-6}$ for a $60 \mathrm{~cm}$ particle track. So the antenna is sensitive to very slow tracks: this is another very important difference with to the usual particle detectors.

\section{Nuclearite search in NAUTILUS and EXPLORER}

The vibrational produced by a nuclearite crossing a bar can be evaluated inserting Eq. (1) in Eq. (2). Considering a nuclearite of mass $M$ and velocity $c \beta$, intersecting orthogonally the center of the NAUTILUS (or EXPLORER) bar we have:

$$
\Delta E[\text { Kelvin }]=10.7\left(\frac{\beta \theta(M)}{10^{-3}}\right)^{4}
$$

where the factor 10.7 is computed using the thermo acoustic parameters measured at $\mathrm{T}=2 \mathrm{~K}[11], \Delta E$ is the energy variation of the bar fundamental mode measured in Kelvin and $\theta(M)=(M / 1.5 n g)^{1 / 3}$ if $\mathrm{M}>1.5 \mathrm{ng}$. Otherwise $\theta(M)=1$. The maximum geometrical acceptance for a nuclearite isotropic distribution is given by $2 \pi S_{t o t}=19.54 \mathrm{~m}^{2} \mathrm{sr}^{-1}$, where $S_{\text {tot }}$ is the bar surface.

In order to select clean events we have applied several cuts to the data. The most important cuts are based on: the gain of the electronic chain, the SQUID locking working point, the seismic monitors, the event shape. We have removed also periods with an high event rates and runs having live-time less than $10 \mathrm{~h}$. This procedure has been applied at the moment only for the NAUTILUS data in the years 2005-2013. The noise cut for NAUTILUS is $T_{\text {eff }}<2.5 \mathrm{mK}$. The total live-time for this data set is 1846 days. The 2003 and 2004 NAUTILUS data are not included because of the high noise. The efficiency of those cuts is verified continuously using the extensive air showers detected by the cosmic ray detector. The energy distribution of the 931 NAUTILUS events surviving the cuts and having $\mathrm{E}>0.1 \mathrm{~K}$ is shown in Fig. 2, together with the result of a Montecarlo simulation for $\beta \theta(M)=0.004$.

For EXPLORER the selection of clean events is in progress and at the moment only a fraction of the previous cuts is applied. The EXPLORER data are used in combination to the 


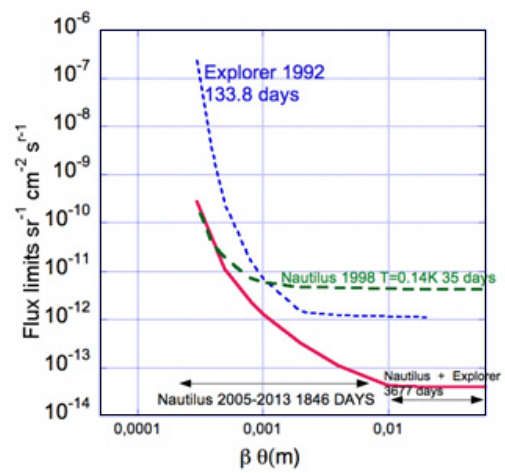

Figure 3. 90\% C.L. isotropic flux upper limits compared to previous results (Explorer) [6]. The bottom curve, in red, shows the results of this analysis. The EXPLORER data are used in combination to the NAUTILUS data only for $\beta \theta(M)>0.01$. Limits from a short run with NAUTILUS at $T=0.14 \mathrm{~K}$, in green, may be interesting because of the different detection mechanism in the superconducting state. The limits are computed using the so called optimum interval method [13], for finding an upper limit of a one-dimensionally distributed signal in the presence of an unknown background. The signal is assumed to have the distribution computed by Montecarlo using Eqs. (2) and (3).

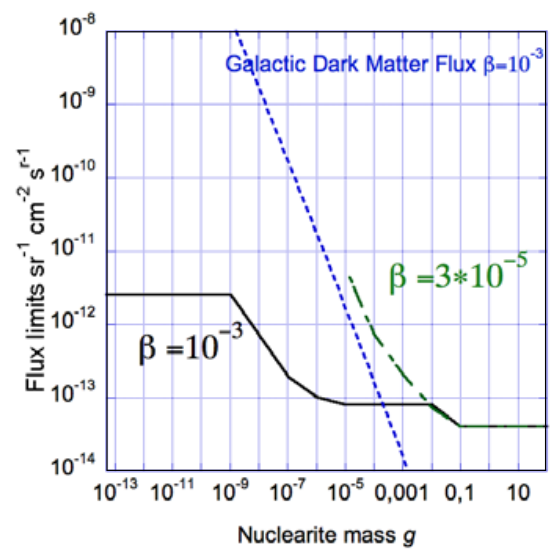

Figure 4. Flux upper limits for $\beta=10^{-3}$ and $\beta=3 \cdot 10^{-5}$ (earth escape velocity) vs mass. The limits are derived from Fig. 3, computing the appropriate $\beta \theta(M)$. For some combination of masses and $\beta$ nuclearites cannot cross the earth, in this case a factor 2 is applied to the limits of Fig. 3. For mass between $5 \cdot 10^{-14} \mathrm{~g}$ (threshold due to the atmosphere) and $10^{-4} \mathrm{~g}$ this limit is significantly smaller than the flux of galactic dark matter for $\beta=10^{-3}$.

NAUTILUS data only for $\beta \theta(M)>0.01$. The noise cut for EXPLORER is $T_{\text {eff }}<5 \mathrm{mK}$ and the live time of the years 2003-2010 is 1831.4 days.

The nuclearite flux upper limits have been computed starting from the energy distributions and is shown in Fig. 3. This figure also shows the limits from a short run with NAUTILUS at $T=0.14 \mathrm{~K}$, with live-time $=35.1$ days. This result may be interesting because of the different detection mechanism in the superconducting state. For $\beta \theta(M)>0.01$, where the background is negligible, the flux upper limit is dominated only by the live-time. Note that in this search the events in coincidence with the cosmic ray detector are removed from the NAUTILUS data. Fast nuclearites could produce light in the EXPLORER scintillators (due to black body emission [4]) and could be confused with a cosmic ray event. Therefore cosmic 
rays are not removed from the EXPLORER data. Finally Fig. 4 shows the upper limits vs the nuclearite mass. For mass between $5 \cdot 10^{-14} \mathrm{~g}$ (threshold due to the atmosphere) and $10^{-4} \mathrm{~g}$ this limit is significantly smaller than the flux of galactic dark matter for $\beta=10^{-3}$.

As shown in Fig. 1 other experiments above sea level using track etch detectors have obtained lower limits, but the detection mechanism is more complicated than the "calibrated" calorimetric technique used in this search.

\section{References}

[1] Gorham, P., W., Phys. Rev. D 86 (2012) 123005/1-8

[2] Lawson K., Phys. Rev. D 83 (2011) 103520/1-9

[3] Witten E., Phys. Rev. D 30 (1984) 272-285

[4] De Rujula, A. and Glashow, S.L., Nature 312 (1984) 734-737

[5] M. Bertaina, A. Cellino and F. Ronga Exp Astron. (2014) doi: 10.1007/ s10686-0149375

[6] Astone P., et al., Phys. Rev. D 47 (1993) 4770-4773

[7] Cecchini, S., et al. (SLIM Coll.), Eur. Phys. J. C 57 (2008) 525-533

[8] Ambrosio, M., et al. (MACRO Coll.), Eur. Phys. J. C 13 (2000) 453-458

[9] Pavalas, G. E., et al., (ANTARES Coll.), Proc. $23^{\text {rd }}$ ECRS, Moscow, 543 (2012)

[10] P. Astone et al., Astropart. Phys. 7 (1997) 231

[11] M. Bassan et al., Nucl. Instrum. Meth. A 659 (2011) 289

[12] Price, P. B., Phys. Rev. D 38 (1988) 3813-3814

[13] S. Yellin, arXiv:0709.2701 [physics.data-an] 